\title{
SEPARATING POINTS FROM CLOSED CONVEX SETS OVER ORDERED FIELDS AND A METRIC FOR $\widetilde{R}^{n}$
}

\author{
ROBERT O. ROBSON
}

\begin{abstract}
Let $R$ be an arbitrary ordered field, let $\bar{R}$ be a real closure, and let $\widetilde{R}$ and $\widetilde{R}^{n}$ denote the real spectra of $\bar{R}[X]$ and $\bar{R}\left[X_{1}, \ldots, X_{n}\right]$. We prove that a closed convex subset in $R^{n}$ may be separated from a point not in it via a continuous "linear" functional taking values in $\widetilde{R}$ and that there is a $\widetilde{R}$-valued metric on $\widetilde{R}^{n}$. The methods rely on the ultrafilter interpretation of points in $\widetilde{R}^{n}$ and on the existence of suprema and infima of sets in $\widetilde{R}$.
\end{abstract}

\section{INTRODUCTION}

A basic result which uses the completeness and order relation on $\mathbb{R}$ is Theorem $\mathbf{I}$ (for $\mathbb{R}$ ). Let $C \subset \mathbb{R}^{n}$ be a closed convex set and let $\mathbf{p} \in R^{n} \backslash C$. Then there exists a linear function

$$
L(\mathbf{x})=\mathbf{a} \cdot(\mathbf{x}-\mathbf{b})
$$

such that $L(\mathbf{p})<0$ and $L(\mathbf{q})>0$ for all $\mathbf{q} \in C$.

Theorem I remains true if $\mathbb{R}^{n}$ is replaced by a locally convex real vector space and $\mathbf{p}$ is replaced by an arbitrary compact set [5,3.3, Satz 4$]$ but we are more interested in the remark that the statement of Theorem I makes sense if $\mathbb{R}$ is replaced by any ordered field $R$. It is false as stated for every $R \neq \mathbb{R}$, but if we replace $L$ by an $\widetilde{R}$-valued linear function where $\widetilde{R}:=\operatorname{Sper} R[X]$ is the real spectrum of $R[X]$, we obtain

Theorem I. Let $R$ be any ordered field, let $C \subset R^{n}$ be a closed convex set, and let $\mathbf{p} \in \mathbb{R}^{n} \backslash C$. Then there exists a continuous $\widetilde{R}$-valued linear function $L: R^{n} \rightarrow \widetilde{R}$ such that $L(\mathbf{p})<0$ and $L(\mathbf{q})>0$ for all $\mathbf{q} \in C$.

In order to prove Theorem I for general $R$, we let $\bar{R}$ be a real closure of $R$ and extend the Euclidean norm to the space

$$
\widetilde{R}^{n}:=\operatorname{Sper} \bar{R}\left[X_{1}, \ldots, X_{n}\right],
$$

which may be thought of as consisting of those points in Sper $R\left[X_{1}, \ldots, X_{n}\right]$ which induce the given order on $R$. This extended norm does not give us

Received by the editors October 25, 1989. Presented at the AMS Meeting \#858, April 20, 1990, in Albuquerque, New Mexico.

1980 Mathematics Subject Classification (1985 Revision). Primary 46A15, 10M15, 52A20.

Key words and phrases. Convex set, real spectrum, ordered fields, real closed field, metric space. 
a metric on $\widetilde{R}^{n}$ because $\widetilde{R}^{n}$ is not equipped with an addition, and we cannot define an inner product on $\widetilde{R}^{n}$ as is discussed after Definition 2 in $\S 3$. However, with virtually no fudging of the definitions, we are able to prove

Theorem II. Let $R$ be any ordered field. Then there is a metric

$$
\mu: \widetilde{R}^{n} \times \widetilde{R}^{n} \rightarrow \widetilde{R}
$$

which extends the Euclidean distance function.

The point of this paper, then, is that the real spectrum provides the correct tool for both compactifying and completing general ordered fields with a view towards preserving at least some of the structure familiar from linear analysis over $\mathbb{R}$. We assume a certain degree of familiarity with the spaces $\widetilde{R}$ and $\widetilde{R}^{n}$. In particular we assume that the reader is familiar with

(1) the various types of points in $\widetilde{R}$ for general real closed $R$,

(2) the description given in [7 $\S 2]$

(3) the "ultrafilter theorem" [3] and ultrafilter arguments, and

(4) semialgebraic maps $f: R^{n} \rightarrow R$ and their extensions $\widetilde{f}: \widetilde{R}^{n} \rightarrow \widetilde{R}$.

All of the necessary background is available in [2, Chapter 7], [1], and [7, §2].

The paper is organized as follows: In $\S 1$ we present a simple counterexample showing why Theorem $\mathrm{I}$ is false if we only allow $R$-valued linear functions, and in $\S 2$ we present an elementary proof of Theorem $I$ for $\mathbb{R}$. In $\S 3$ we explain what we mean by a $\widetilde{R}$-valued linear function and prove a continuity result for these functions. In $\S 4$ we isolate two results needed to modify the proof given in $\S 2$, and in $\S 5$ we prove Theorem I for general $R$. In $\S 6$ we generalize the notion of "slices" from [7] to one called "rips". Rips allow us to add points in $\widetilde{R}^{n}$ and to compare the resulting sums so that we may interpret the triangle inequality. In $\S 7$ we define $\mu$ and prove Theorem II.

We point out right away that, for the purposes of our proofs, we will assume that $R$ is real closed. This is no loss of generality since if $\bar{R}$ is a real closure of the ordered field $R$ and $n \geq 1$, then $\widetilde{R}^{n}={\widetilde{\bar{R}^{n}}}^{n}$ as we have defined it.

The motivation for this paper came from Bruce Reznick who conjectured that Theorem I (as stated for $\mathbb{R}$ ) failed for nonarchimedean $R$ during a talk on blenders in May, 1989. A few weeks later Tom Craven pointed out the counterexample in $\S 1$ for the case $R=\mathbb{Q}$. The author wishes to thank both Reznick and Craven for their inspiration and an anonymous referee for suggesting some clarifications and simplifications which have been incorporated.

\section{A COUNTEREXAMPLE}

Suppose $R \neq \mathbb{R}$ and that $\alpha$ is a (finite) Dedekind cut of $R$ which is not represented by an element of $R$. In keeping with notation from [7, §2], we will represent $\alpha$ by its left set $\alpha_{l}$ and its right set $\alpha_{r}$. Thus $\alpha_{l}$ and $\alpha_{r}$ are

\footnotetext{
We use the word "interval" to include singletons, half-infinite, half-open, closed, infinite, and half-infinite intervals-in short, any semialgebraically connected subset of $R$.
} 
two nonempty open half-infinite intervals ${ }^{1}$ with the properties that $\alpha_{l} \leq \alpha_{r}$, meaning that $x \in \alpha_{l}, y \in \alpha_{r} \Rightarrow x \leq y$, and that $\alpha_{l} \cup \alpha_{r}=R$. In the case at hand, $\alpha_{l}<\alpha_{r}$.

Now consider the upper half-plane $H \subset R^{2}$ whose boundary is the nonexistent line $x_{2}=\alpha x_{1}$. Thus $H$ consists of all points $\left(0, x_{2}\right)$ with $x_{2} \geq 0$ plus all $\left(x_{1}, t x_{1}\right) \in R^{2}$ such that

$$
x_{1} \geq 0 \text { and } t \in \alpha_{r} \text { or } \quad x_{1} \leq 0 \text { and } t \in \alpha_{l} .
$$

$H$ is closed and convex, but there is no way to separate $H$ from any $\mathbf{p} \notin H$ using a line. To see this, suppose $t \in \alpha_{r}$ and consider the line $x_{2}=t x_{1}+b$. Using the fact that $\alpha_{r}$ has no left endpoint, we may choose $x_{1}$ positive and large enough so that $t x_{1}+b=t^{\prime} x_{1}$ with $t^{\prime}$ still in $\alpha_{r}$. Thus $\left(x_{1}, t x_{1}+b\right)=$ $\left(x_{1}, t^{\prime} x_{1}\right) \in H$. The same idea works for $t \in \alpha_{l}$ and the lines $x_{1}=c$. In other words, every line intersects $H$. This shows that Theorem I as stated for $\mathbb{R}$ needs some modification for other $R$.

\section{A PROOF OF THEOREM I FOR $\mathbb{R}$}

This section contains a very simple proof of Theorem I for $\mathbb{R}$ based upon the following lemma:

Lemma 1. Let $\mathbf{p}, \mathbf{q} \in \mathbb{R}^{n}$ and suppose that $\mathbf{p} \cdot \mathbf{q}<\|\mathbf{p}\|^{2}$. Then for all sufficiently small $t>0$ we have

$$
\|t \mathbf{q}+(1-t) \mathbf{p}\|^{2}<\|\mathbf{p}\|^{2}
$$

Proof. Use the fact that

$$
\|t \mathbf{q}+(1-t) \mathbf{p}\|^{2}-\|\mathbf{p}\|^{2}=t\left\{t\left(\|\mathbf{q}\|^{2}+\|\mathbf{p}\|^{2}-2 \mathbf{q} \cdot \mathbf{p}\right)+2\left(\mathbf{q} \cdot \mathbf{p}-\|\mathbf{p}\|^{2}\right)\right\} .
$$

Proof of Theorem I over $\mathbb{R}$. We may assume that $\mathbf{p}=\mathbf{0}$. Let $\mathbf{a} \in C$ be a point with minimal distance to $\mathbf{0}$, i.e., with minimal Euclidean norm. The existence of a is implied by the completeness of $\mathbb{R}^{n}$. Lemma 1 and convexity imply that

$$
\mathbf{a} \cdot \mathbf{x} \geq\|\mathbf{a}\|^{2}
$$

for all $\mathbf{x} \in C$. Thus $L(\mathbf{x})=\mathbf{a} \cdot(\mathbf{x}-\mathbf{a} / 2)$ is the sought after function.

In $\S 5$ we carry out this proof for general $R$, but first we need to compactify the domain $R^{n}$, complete the range $R$, and define new linear functions. This is done via the real spectrum, starting in the next section.

\section{3. $\widetilde{R}$-VALUED LINEAR FUNCTIONS}

The semialgebraic subsets of $R^{n}$ form a boolean algebra and $\widetilde{R}^{n}$ may be defined as the set of ultrafilters of this algebra. (Remember, we are assuming that $R$ is real closed.) We will use this definition exclusively and denote points of $\widetilde{R}^{n}$ by lower case Greek letters. The topology on $\widetilde{R}^{n}$ is that generated by 
the sets

$$
\widetilde{U}:=\left\{\alpha \in \widetilde{R}^{n} \mid U \in \alpha\right\}
$$

for open semialgebraic sets $U \subset R^{n}$. $\widetilde{R}^{n}$ is quasicompact but not Hausdorff. If $\beta$ is in the closure of $\alpha$ we say that $\alpha$ specializes to $\beta$ and write $\alpha \rightarrow \beta$. The concepts of closed and bounded for points are defined by

Definition 1. A point $\alpha \in \widetilde{R}^{n}$ is closed if it has no proper specializations and bounded if it contains a bounded set.

If $A \subseteq R^{n}$ and $B \subseteq R$ are semialgebraic sets, and $f$ is a map with semialgebraic graph, then $f(A)$ and $f^{-1}(B)$ are also semialgebraic [2]. It is easy to verify that

$$
\{f(A) \mid A \in \alpha\}
$$

generates an ultrafilter if $\alpha \in \widetilde{R}^{n}$ is an ultrafilter. Thus $f$ induces a function $\widetilde{f}: \widetilde{R}^{n} \rightarrow \widetilde{R}$ where $\widetilde{f}(\alpha)$ is defined to be the ultrafilter in $\widetilde{R}$ generated by (4). If $f$ is also continuous, then $\tilde{f}$ is continuous as a function from $\widetilde{R}^{n}$ to $\widetilde{R}$. Details appear in [1], [2, 7.2.8], and [2, 7.3].

As a particular example of this, fix $\mathbf{x} \in R^{n}$ and consider the dual linear function

$$
L_{\mathbf{x}}(\mathbf{y}):=\mathbf{x} \cdot \mathbf{y}
$$

Then $L_{\mathbf{x}}$ is a continuous semialgebraic function, so we may extend it to a function

$$
\widetilde{L}_{\mathbf{x}}: \widetilde{R}^{n} \rightarrow \widetilde{R}
$$

whose value at the ultrafilter $\alpha$ is computed by considering each semialgebraic subset $A \in \alpha$ and dotting each point in $A$ with $\mathbf{x}$. This produces a semialgebraic subset of $R$, and the set of these sets forms an ultrafilter $\widetilde{L}_{\mathbf{x}}(\alpha)$ in $\widetilde{R}$.

This process may be dualized to produce a function

$$
L_{\alpha}: R^{n} \rightarrow \widetilde{R}, \quad \mathbf{x} \mapsto L_{\mathbf{x}}(\alpha) .
$$

Definition 2. An $\widetilde{R}$-valued linear function is a map $L: R^{n} \rightarrow \widetilde{R}$ of the form $L(\mathbf{x})=L_{\alpha}(\mathbf{x}-\mathbf{b})$ with $L_{\alpha}$ as in (7) and $\mathbf{b} \in R^{n}$.

We remark that extending $L_{a}$ to all of $\widetilde{R}^{n}$, i.e., defining an inner product on $\widetilde{R}^{n}$, is too much to ask for. Indeed, for $n=1$ this would define a multiplication on $\widetilde{R}$, and this is hopeless if $R$ is nonarchimedean. To see why, let $\eta$ be the ultrafilter of semialgebraic sets which span the gap between the positive infinitesimals and the positive noninfinitesimal elements (with respect to $\mathbb{Q}$ ), and let $\zeta$ be the ultrafilter spanning the gap between the positive finite and the positive infinite elements. The product of any set from $\eta$ with any set from $\zeta$ contains all positive, finite, noninfinitesimal elements. Thus " $\eta \cdot \zeta$ " is contained in an infinite number of ultrafilters. 
Also, the function $L_{\eta}(x)$ jumps from $\eta$ to $\zeta$ as the argument $x \in R$ crosses the gap $\zeta . L_{\eta}$ is therefore not a slice [7] and certainly cannot be extended to a continuous function $\widetilde{R}^{n} \rightarrow \widetilde{R}$. In general, $L_{\alpha}$ need not even be continuous as function from $R^{n} \rightarrow \widetilde{R}$ as can be seen by taking $n=1$ and $\alpha=+\infty$. But we do have

Proposition 1. If $\alpha$ is closed and bounded, then the function $L$ in Definition 2 is continuous. For any $\alpha$ we have the following "sublinearity" property: If $s_{1} \leq L(\mathbf{x}) \leq s_{2}$ and $t_{1} \leq L(\mathbf{y}) \leq t_{2}$ for $s_{1}, s_{2}, t_{1}, t_{2} \in R$, then $s_{1}+s_{2} \leq$ $L(\mathbf{x}+\mathbf{y}) \leq t_{1}+t_{2}$.

Proof. We may assume that $L=L_{\alpha}$. A subbasic open set in $\widetilde{R}$ consists of the set $\widetilde{I}$ of ultrafilters containing an open interval $I=(a, b) \subset R$. We have

$$
L_{\alpha}(\mathbf{x}) \in \widetilde{I} \text { if and only if } \mathbf{x} \cdot A \subseteq I \text { for some } A \in \alpha .
$$

Now, if $L_{\alpha}(\mathbf{x}) \in \widetilde{I}$, there must be some closed bounded $B \in \alpha$ such that $\mathbf{x} \cdot B$ is a finite union of (necessarily closed and bounded) subintervals of $(a, b)$. For otherwise every closed bounded set $B \in \alpha$ would contain points in the semialgebraic set

$$
K:=\{\mathbf{y} \mid \mathbf{x} \cdot \mathbf{y} \leq a \text { or } \mathbf{x} \cdot \mathbf{y} \geq b\} .
$$

Since every closed semialgebraic set in $\alpha$ contains a closed bounded semialgebraic set in $\alpha$, the set of $B \cap K$ with $B \in \alpha$ and $B$ closed would then have the finite intersection property, from which the existence of a specialization $\beta$ of $\alpha$ with $L_{\beta}(\mathbf{x}) \notin(a, b)$ would follow. Since $L_{\beta}(\mathbf{x}) \neq L_{\alpha}(\mathbf{x})$, we see $\beta \neq \alpha$, contradicting the assumption that $\alpha$ is closed.

We have seen that there is a closed bounded $B \in \alpha$ such that $\mathbf{x} \cdot B \subseteq[c, d]$ with

$$
a<c<d<b .
$$

Using (9) and a bound on the norm of points in $B$ it is straightforward to find an $\varepsilon>0$ so that $\mathbf{z} \cdot B \subseteq I$ whenever $\|\mathbf{x}-\mathbf{z}\|<\varepsilon$. This implies $L_{\alpha}(\mathbf{z}) \in \widetilde{I}$ whenever $\|\mathbf{x}-\mathbf{z}\|<\varepsilon$ and establishes continuity.

For the last statement, we note that there is a set $A \in \alpha$ such that for every $\mathbf{a} \in A$ we have $s_{1} \leq \mathbf{a} \cdot \mathbf{x} \leq s_{2}$ and $t_{1} \leq \mathbf{a} \cdot \mathbf{y} \leq t_{2}$ and hence $s_{1}+s_{2} \leq \mathbf{a} \cdot(\mathbf{x}+\mathbf{y}) \leq$ $t_{1}+t_{2}$.

Finally, we point out that if $R$ is an arbitrary ordered field and $\alpha=\mathbf{x}$ is a rational point in $\bar{R}^{n}$ over a real closure of $R$, then $L_{\alpha}$ is just the restriction of the dot product with $\mathbf{x}$ to $R^{n}$.

\section{SOME GROUNDWORK}

The purpose of this section is to recall some key results on $\widetilde{R}$ from [7] and to isolate a slightly technical but trivial lemma on abstract functions.

In [7, §2] it is shown that the points $\alpha \in \widetilde{R}$ for $R$ real closed may be represented as pairs $\left(\alpha_{l}, \alpha_{r}\right)$ of subsets of $R$, called slices, satisfying $\alpha_{l} \leq \alpha_{r}$ 
and $\alpha_{l} \cup \alpha_{r}=R$. Specifically, for a point $x \in R$ we have

$$
\begin{aligned}
& x \in \alpha_{l} \text { if and only if }(-\infty, x] \in \alpha, \\
& x \in \alpha_{r} \text { if and only if }[x,+\infty) \in \alpha .
\end{aligned}
$$

The crucial result for us is

Proposition 2. $\widetilde{R}$ is totally ordered with

$$
\alpha \leq \beta \text { if and only if } \alpha_{l} \subseteq \beta_{l} \text { and } \beta_{r} \subseteq \alpha_{r} .
$$

Every subset of $\widetilde{R}$ has both a supremum and an infimum in $\widetilde{R}$.

Proof. [7, §2].

The technical result we need, which we state in more generality than necessary, is a souped-up version of the result that the extension of a continuous semialgebraic function to $\widetilde{R}^{n}$ assumes a minimum on a closed subset: Let $K \subseteq \widetilde{R}^{n}$ be closed and let $f: R^{n} \rightarrow R$ be a continuous semialgebraic function. Let

$$
\lambda:=\inf _{\alpha \in K} \widetilde{f}(\alpha) .
$$

In addition, let $\left\{g_{s}\right\}_{s \in \mathcal{S}}$ be a family of continuous semialgebraic functions from $R^{n}$ to $R$ and let $\left\{\kappa_{s}\right\}_{s \in \mathscr{S}}$ be a family of closed points in $\widetilde{R}$ such that for any finite subset $s_{1}, \ldots, s_{m} \in \mathscr{S}$ and any $\varepsilon \in R$ with $\varepsilon \geq \lambda$ there are points $\alpha \in K$ such that simultaneously $\tilde{f}(\alpha) \leq \varepsilon$ and $\widetilde{g}_{s_{i}}(\alpha) \geq \kappa_{s_{i}}$ for $i=1, \ldots, m$.

Lemma 2. Under the hypotheses just given there is a closed point $\zeta \in K$ with $\widetilde{f}(\zeta)=\lambda$ and $g_{s}(\zeta) \geq \kappa_{s}$ for all $s \in \mathscr{S}$.

Proof. The sets

$$
K_{\varepsilon, s_{1}, \ldots, s_{m}}:=\left\{\alpha \in K \mid \widetilde{f}(\alpha) \leq \varepsilon \text { and } \widetilde{g}_{s_{i}}(\alpha) \geq \kappa_{s_{i}} \text { for } i=1, \ldots, m\right\}
$$

are closed and have the finite intersection property. Therefore there is a closed point $\zeta$ in their intersection.

\section{Proof of Theorem I}

Let $C \subset R^{n}$ be closed and convex and assume that $0 \notin C$ with the intention of proving Theorem I. Let

$$
N^{2}(\mathbf{x})=\|\mathbf{x}\|^{2}=\sum_{i=1}^{n} x_{i}^{2}
$$

be the square of the Euclidean norm, which is a continuous semialgebraic function. The following is a version of Lemma 1 from $\S 2$ :

Lemma 3. Let $\mathbf{p}$ and $\mathbf{q}_{1}, \ldots, \mathbf{q}_{m}$ be finitely many points in $C$. Then there is a point $\mathbf{a} \in C$ such that $N(\mathbf{a}) \leq N(\mathbf{p})$ and such that for $i=1, \ldots, m$ we have

$$
\mathbf{q}_{i} \cdot \mathbf{a} \geq N^{2}(\mathbf{a})
$$


Proof. We might as well assume that $C$ is the convex hull of the $\mathbf{q}_{i}$. This is a closed bounded semialgebraic set, so there is a point $a$ in it of minimal norm $[2,2.5 .8]$. The calculation used to prove Lemma 1 is valid over any $R$, from which (12) follows.

The same argument works in the case of an arbitrary closed bounded semialgebraic set $C$ and (using the fact that a semialgebraic function achieves a maximum on $C$ as well) yields

Lemma 4. If $C$ is a closed bounded semialgebraic set in $R^{n}$ which does not contain 0 , then there is a vector $\mathbf{x} \in R^{n}$ such that $\mathbf{x} \cdot C$ is contained in a closed interval $[a, b]$ with $0<a \leq b<\infty$.

Proof of Theorem I. Let $K$ be the closure of $C$ in $\widetilde{R}^{n}$ and let

$$
\lambda:=\inf _{\mathbf{q} \in C} N(\mathbf{q}) \text {. }
$$

Since $C$ is closed, we have $0^{+}<\lambda$, so we may choose a $z \in R$ with $0<$ $z \leq \lambda$. We now consider the semialgebraic function $N$, the family $\left\{L_{\mathbf{q}}\right\}_{\mathbf{q} \in C}$ of semialgebraic functions, and the constant family $\kappa_{\mathrm{q}}=z^{2}$. If $\varepsilon \geq \lambda$, there is a point $\mathbf{p} \in C$ with $N(\mathbf{p}) \leq \varepsilon$. Lemma 3 says that this setup satisfies the hypotheses of Lemma 2 , so we find a closed $\zeta \in K$ such that

$$
\widetilde{N}(\zeta)=\lambda \text { and } \widetilde{L}_{\mathbf{q}}(\zeta) \geq z^{2} \text { for all } \mathbf{q} \in C .
$$

Let $\widetilde{L}_{\zeta}$ be defined as in (7). From (13) we see both that $\zeta$ is bounded and that $\tilde{L}_{\zeta}(\mathbf{q}) \geq z^{2}$ for $\mathbf{q} \in C$. By Proposition 1 the function

$$
L(\mathbf{x})=\widetilde{L}_{\zeta}(\mathbf{x}-\mathbf{b})
$$

fulfills the requirements of Theorem I for any $\mathbf{b}$ with $-z^{2} / 2<\widetilde{L}_{\zeta}(-\mathbf{b})<0$.

To see that such a b exists, note that $\zeta$ contains a closed bounded $B$ which does not contain 0 . We now apply Lemma 4 to $B$ and let $\mathbf{b}=-\frac{z^{2}}{2 b} \mathbf{x}$ with $\mathbf{x}$ and $b$ as in the lemma.

\section{SLICES AND RIPS}

In the description of $\widetilde{R}$ from [7], the set $\alpha_{l}$ is called the left set of $\alpha$ and consists of all $x \in R$ with $x \leq \alpha$, while $\alpha_{r}$ is called the right set of $\alpha$ and consists of all $x$ with $\alpha_{r} \leq x$. If we try to add two points $\alpha$ and $\beta$ by adding their left and right sets, we see that

$$
\alpha_{l}+\beta_{l} \leq \alpha_{r}+\beta_{r}
$$

and also that

$$
\begin{aligned}
& x \in \alpha_{l}+\beta_{l} \text { and } y<x \Rightarrow y \in \alpha_{l}+\beta_{l}, \\
& x \in \alpha_{r}+\beta_{r} \text { and } y>x \Rightarrow y \in \alpha_{r}+\beta_{r},
\end{aligned}
$$

but in general there is a gap between the set sums in (14). For example, the point 2 is in neither the sum of the left sets nor in the sum of the right sets of " $1^{-}+1^{+} "$. In the nonarchimedean case entire intervals may be left out. 
For this reason we are led to consider more general pairs

$$
\zeta=\left(\zeta_{l}, \zeta_{r}\right)
$$

of left and right sets and define

Definition 3. A rip is a pair (15) satisfying

$$
\begin{gathered}
\zeta_{l} \leq \zeta_{r}, \\
x \in \zeta_{l} \text { and } y<x \Rightarrow y \in \zeta_{l}, \text { and } \\
x \in \zeta_{r} \text { and } y>x \Rightarrow y \in \zeta_{r} .
\end{gathered}
$$

Thus points in $\widetilde{R}$ are special types of rips. If $\zeta$ and $\xi$ are rips, we define

$$
\zeta+\xi=\left(\zeta_{l}+\xi_{l}, \zeta_{r}+\xi_{r}\right)
$$

which is again a rip. Next, we borrow from John Conway's philosophy (used to define the ordering on the surreal numbers in [JC, Chapter 1]) and define $\zeta \leq \xi$ unless there is an obstruction to this inequality. An obstruction is a point $x \in R$ with either $\xi<x \leq \zeta$ or $\xi \leq x<\zeta$. We interpret $\xi \leq x$ to mean that $x \in \xi_{r}$ and $\xi<x$ to mean that $x \in \xi_{r} \backslash \xi_{l}$. There is no such obstruction $x$ if

$$
\zeta_{l} \cap \xi_{r} \backslash \xi_{l}=\zeta_{l} \cap \xi_{r} \backslash \zeta_{r}=\varnothing,
$$

which may be rephrased as

Definition 4. Let $\zeta$ and $\xi$ be two rips. We define $\zeta \leq \xi$ if and only if and $\left(\zeta_{l} \cap \xi_{r}\right) \subseteq\left(\zeta_{r} \cap \xi_{l}\right)$.

Proposition 3. The " $\leq$ "-relation on rips extends the total ordering on $\widetilde{R}$. Given two rips $\zeta$ and $\xi$, either $\zeta \leq \xi$, or $\xi \leq \zeta$ or both.

Proof. The first statement follows from the development of Definition 4 and can be checked by looking at cases. To prove the second statement, we need to exclude the possibility that there are $x, y \in R$ with

$$
\begin{aligned}
& x \in \zeta_{l} \cap \xi_{r} \text { and } x \notin \zeta_{r} \cap \xi_{l}, \\
& y \in \zeta_{r} \cap \xi_{l} \text { and } y \notin \zeta_{l} \cap \xi_{r} .
\end{aligned}
$$

But if (18) holds, then $x \in \zeta_{l}$ and $y \in \zeta_{r}$ imply that $x \leq y$, while $x \in$ $\xi_{r}$ and $y \in \xi_{l}$ imply that $x \geq y$. Hence $x=y$, but now (18) is clearly contradictory.

\section{THE METRIC $\mu$}

To define $\mu$, consider $\alpha, \beta \in \widetilde{R}^{n}$. If $A \in \alpha$ and $B \in \beta$, we define

$$
d(A, B):=\inf _{\substack{\mathbf{a} \in A \\ \mathbf{b} \in B}}\|\mathbf{a}-\mathbf{b}\| .
$$

Note that the infimum in (19) is to be taken not in $R$ (where it need not exist) but in $\widetilde{R}$. We then go on to define 


$$
\mu(\alpha, \beta):=\sup _{\substack{A \in \alpha \\ B \in \beta}} d(A, B)
$$

where the supremum is taken in $\widetilde{R}$ as well. Interpreting the values of $\mu$ as rips for the triangle inequality, we have:

Theorem II. The function $\mu: \widetilde{R}^{n} \times \widetilde{R}^{n} \rightarrow \widetilde{R}$ is a positive definite symmetric function satisfying the triangle inequality. Moreover, if $\mathbf{a}, \mathbf{b} \in R^{n} \subset \widetilde{R}^{n}$, then $\mu(\mathbf{a}, \mathbf{b})=\|\mathbf{a}-\mathbf{b}\|$.

Proof. Symmetry is obvious from the definition as is the fact that $\mu(\alpha, \beta) \geq 0$. For the rest of the proof we let $\alpha, \beta, \gamma \in \widetilde{R}^{n}$.

Suppose $\mu(\alpha, \beta)=0$. Since $\{x \in R \mid x>0\}$ has the infimum $0^{+}$in $\widetilde{R}$, every set $A \in \alpha$ must have zero distance to every set $B \in \beta$. In other words, $A \cap B \neq \varnothing$ for every $A \in \alpha$ and $B \in \beta$. Thus $\alpha \cup \beta$ is a filter, which implies $\alpha=\beta$ since both are ultrafilters. Thus $\mu$ is positive definite.

The triangle inequality states that

$$
\mu(\alpha, \gamma) \leq \mu(\alpha, \beta)+\mu(\beta, \gamma) .
$$

To verify this we need to show that

$$
\left(\mu(\alpha, \gamma)_{l} \cap\left(\mu(\alpha, \beta)_{r}+\mu(\beta, \gamma)_{r}\right)\right) \subseteq\left(\mu(\alpha, \gamma)_{r} \cap\left(\mu(\alpha, \beta)_{l}+\mu(\beta, \gamma)_{l}\right)\right) .
$$

So suppose there is a point $z$ in the left-hand set given in (21). Since $z \in$ $\mu(\alpha, \beta)_{r}+\mu(\beta, \gamma)_{r}$, there are points $x, y \in R$ such that $x+y=z$ and such that given any $A \in \alpha, B \in \beta$, and $C \in \gamma$ there are points $\mathbf{a} \in A, \mathbf{b} \in B$ and $\mathbf{b}^{\prime} \in B, \mathbf{c} \in C$ with

$$
\|\mathbf{a}-\mathbf{b}\| \leq x \quad \text { and } \quad\left\|\mathbf{b}^{\prime}-\mathbf{c}\right\| \leq y .
$$

Fixing $A, B$, and $C$, consider the set

$$
B^{\prime}:=\{\mathbf{b} \in B \mid d(A,\{\mathbf{b}\}) \leq x\} .
$$

We must have $B^{\prime} \in \beta$, for otherwise its complement would be in $\beta$ and (22) could not hold for any $\mathbf{b} \in B^{\prime}$. Now consider the set

$$
B^{\prime \prime}:=\left\{\mathbf{b}^{\prime} \in B^{\prime} \mid d\left(\left\{\mathbf{b}^{\prime}\right\}, C\right) \leq y\right\} .
$$

Again, we must have $B^{\prime \prime} \in \beta$. But this implies that $d(A, C) \leq z$ for any $A \in \alpha$ and $C \in \gamma$. Thus we conclude that $z \in \mu(\alpha, \gamma)_{r}$.

By assumption, $z \in \mu(\alpha, \gamma)_{l}$. Thus there are sets $A \in \alpha$ and $C \in \gamma$ with $d(A, C) \geq z$. Fix these sets. Suppose there were an $E \in \beta$ with $d(A,\{\mathbf{e}\})<$ $x$ for all $\mathrm{e} \in B$. By intersecting $E$ with $B^{\prime \prime}$ from (23) we would obtain $d(A, C)<z$. Thus the set

$$
E:=\left\{\mathbf{b} \in B^{\prime} \mid d(A,\{\mathbf{b}\})=x\right\}
$$

is in $\beta$, from which we see that $x \in \mu(\alpha, \beta)_{l}$. Similarly, $y \in \mu(\beta, \gamma)_{l}$, and so $z=x+y \in \mu(\alpha, \beta)_{l}+\mu(\beta, \gamma)_{l}$, establishing (21). 
Some examples. In order to illustrate $\mu$, we state (without proof) some values in a few simple cases. First, a table of values for pairs of points in $\widetilde{R}$ with the first point specializing to 1 and the second to 2 :

$$
\begin{aligned}
\mu\left(1^{-}, 2^{-}\right) & =1^{-}, & \mu\left(1,2^{-}\right) & =1^{-}, & \mu\left(1^{+}, 2^{-}\right) & =1^{+}, \\
\mu\left(1^{-}, 2\right) & =1^{+}, & \mu(1,2) & =1^{-}, & \mu\left(1^{+}, 2\right) & =1^{-}, \\
\mu\left(1^{-}, 2^{+}\right) & =1^{+}, & \mu\left(1,2^{+}\right) & =1^{+}, & \mu\left(1^{+}, 2^{+}\right) & =1^{-} .
\end{aligned}
$$

For a slightly more complicated case, consider the two-dimensional point $\alpha \in$ $\widetilde{\mathbb{R}}^{2}$ consisting of all semialgebraic subsets $A$ containing a set of the form $\left\{\left(x, e^{x}\right) \mid 0<x<\varepsilon\right\}$ for some $\varepsilon>0$. Let $\beta$ be the one-dimensional point consisting of all semialgebraic sets containing some piece of the algebraic half-branch $y=0, x>0$ at $(0,0)$. Then $\mu(\alpha, \beta)=1^{+}$.

In order to clarify the nature of $\mu$, we point out

Proposition 4. Suppose $\alpha, \beta \in \widetilde{R}^{n}$ and $\alpha$ is bounded. Then $\mu(\alpha, \beta)=0^{+}$if and only if $\alpha$ and $\beta$ are distinct but have a common specialization.

Proof. Suppose $\mu(\alpha, \beta)=0^{+}$. Let $A \in \alpha$ be bounded, let $B \in \beta$ be arbitrary, and let $\bar{A}$ and $\bar{B}$ be their closures in $R^{n}$. If we fix $\mathbf{x}$, then the function of $\mathbf{x}$ which measures the distance to $\bar{B}$ is a continuous semialgebraic function [2 2.5.8]. This takes a minimum on the closed bounded set $\bar{A}$. If $\mu(\alpha, \beta)=0^{+}$, this minimum cannot be strictly positive, so $\bar{A} \cap \bar{B} \neq \varnothing$. We conclude that

$$
\{\bar{A} \cap \bar{B} \mid A \in \alpha, B \in \beta\}
$$

has the finite intersection property and is contained in at least one ultrafilter $\gamma$. Since any closed set in $\alpha$ is in $\gamma, \alpha \rightarrow \gamma$. Similarly, $\beta \rightarrow \gamma$.

If $\alpha$ and $\beta$ have no common specialization, we can find disjoint closed sets $A \in \alpha$ and $B \in \beta$ [7], and since we may choose $A$ to be bounded, it is immediate that $\mu(\alpha, \beta) \geq d(A, B)=x>0$ for some $x \in R$, so $\mu(\alpha, \beta)>$ $0^{+}$.

\section{REFERENCES}

1. E. Becker, On the real spectrum of a ring and its application to semialgebraic geometry, Bull. Amer. Math. Soc. 15 (1986), 19-60.

2. J. Bochnak, M. Coste, and M.-F. Roy, Géométrie algébrique réelle, Ergebnisse der Mathematik und ihrer Grenzgebiete, Springer-Verlag, New York, Berlin, and Heidelberg, 1987.

3. L. Bröcker, Real spectra and distributions of signatures, Géométrie Algébrique Réelle et Formes Quadratiques, Lecture Notes in Math., vol. 959, Springer-Verlag, New York, Berlin, and Heidelberg, 1982.

4. J. H. Conway, On numbers and games, Academic Press, London and New York, 1976.

5. J. Marti, Konvexe Analysis, Lehrbücher und Mono. aus dem Gebiete der exakten Wissenschaften, Mathematische Reihe 54, Birkhaüser, Basel und Stuttgart, 1977. 
6. S. Prieß-Crampe, Angeordnete Strukturen: Gruppen, Körper, projektive Ebenen, Ergebnisse der Mathematik und ihrer Grenzgebiete 98, Springer-Verlag, New York, Berlin, and Heidelberg, 1983.

7. R. Robson, Slices: Functions for abstract real analysis, real analytic and algebraic geometry, (M. Galbiati and A. Tognoli, eds.), Lecture Notes in Math., vol. 1420, Springer, 1990, pp. 206-222.

Department of Mathematics, Oregon State University, Corvallis, Oregon 97331-4605 